\title{
Reduced expression of glucocorticoid-inducible genes GILZ and SGK-1: high IL-6 levels are associated with reduced hippocampal volumes in major depressive disorder
}

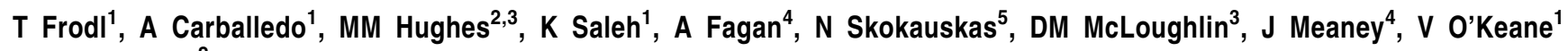 \\ and TJ Connor ${ }^{2}$
}

Neuroplasticity may have a core role in the pathophysiology of major depressive disorder (MDD), a concept supported by experimental studies that found that excessive cortisol secretion and/or excessive production of inflammatory cytokines impairs neuronal plasticity and neurogenesis in the hippocampus. The objective of this study was to examine how changes in the glucocorticoid and inflammatory systems may affect hippocampal volumes in MDD. A multimodal approach with structural neuroimaging of hippocampus and amygdala, measurement of peripheral inflammatory proteins interleukin (IL)-6 and C-reactive protein (CRP), glucocorticoid receptor (GR) mRNA expression, and expression of glucocorticoid-inducible genes (glucocorticoid-inducible genes Leucin Zipper (GILZ) and glucocorticoid-inducible kinase-1 (SGK-1)) was used in 40 patients with MDD and 43 healthy controls (HC). Patients with MDD showed smaller hippocampal volumes and increased inflammatory proteins IL-6 and CRP compared with HC. Childhood maltreatment was associated with increased CRP. Patients with MDD, who had less expression of the glucocorticoid-inducible genes GILZ or SGK-1 had smaller hippocampal volumes. Regression analysis showed a strong positive effect of GILZ and SGK-1 mRNA expression, and further inverse effects of IL- 6 concentration, on hippocampal volumes. These findings suggest that childhood maltreatment, peripheral inflammatory and glucocorticoid markers and hippocampal volume are interrelated factors in the pathophysiology of MDD. Glucocorticoid-inducible genes GILZ and SGK-1 might be promising candidate markers for hippocampal volume changes relevant for diseases like MDD. Further studies need to explore the possible clinical usefulness of such a blood biomarker, for example, for diagnosis or prediction of therapy response.

Translational Psychiatry (2012) 2, e88; doi:10.1038/tp.2012.14; published online 13 March 2012

\section{Introduction}

Major depressive disorder (MDD) is a serious psychiatric disorder with a high relapse rate and lifetime prevalence of $15 \% .{ }^{1}$ Despite the experimental findings involving neuroplasticity in the pathophysiology of MDD, ${ }^{2}$ to date this work has not been translated into the clinical setting in terms of elucidating a causal role for stress and inflammatory cytokines in mediating hippocampal changes. In the clinic, it is well established that depressed patients hypersecrete cortisol, ${ }^{3}$ have impaired glucocorticoid receptor (GR) functioning, ${ }^{4}$ have increased circulating concentrations of inflammatory cytokines ${ }^{5}$ and C-reactive protein $(\mathrm{CRP})^{6}$ and have reduced hippocampal volumes. ${ }^{7}$ It has been shown that hippocampal volume reduction is associated with exposure to stress ${ }^{7}$ and that it is influenced by genetic variation. $^{8}$
The first study that assessed the association between hippocampal volumes and cortisol measures was published by Lupien et al. ${ }^{9}$ This longitudinal study conducted over 5 years in older adults demonstrated a correlation over time between cortisol levels and hippocampal volume changes. The total hippocampal volume of six subjects with increasing or high cortisol levels was significantly reduced by $14 \%$ in comparison with that of five subjects with decreasing/moderate cortisol levels and the degree of hippocampal atrophy correlated strongly with both the degree of cortisol elevation over time and current basal cortisol levels. Since then, there is some further evidence that reduced hippocampal volumes are associated with higher continuous cortisol secretion during the day ${ }^{10-12}$ and with abnormal blunted cortisol responses during awakening or during stress tests. ${ }^{13-16}$ However, data are inconsistent ${ }^{17,18}$ and heterogeneous, even between the

\footnotetext{
${ }^{1}$ Department of Psychiatry and Institute of Neuroscience, Adelaide and Meath Hospital incorporating the National Children's Hospital and St James' s Hospital, University Dublin, Trinity College, Dublin, Ireland; ' 2 Department of Physiology, Neuroimmunology Research Group, School of Medicine and Trinity College Institute of Neuroscience, Trinity College, Dublin, Ireland; ${ }^{3}$ Department of Psychiatry and Trinity College Institute of Neuroscience, Trinity College Dublin, St Patrick's University Hospital, Dublin, Ireland; ${ }^{4}$ Centre of Advanced Medical Imaging and Department of Radiology, St James's Hospital, Dublin, Ireland and ${ }^{5}$ Department of Psychiatry, Child and Adolescent Psychiatry, University Dublin, Trinity College, Dublin, Ireland

Correspondence: Professor T Frodl, Department of Psychiatry and Institute of Neuroscience, Lloyd Building, Trinity College Dublin, Dublin 2, Ireland.

E-mail: thomas.frodl@ @tcd.ie

Keywords: childhood maltreatment; gene expression; glucocorticoid; inflammation; major depressive disorder; neuroimaging

Received 22 November 2011; revised 23 January 2012; accepted 29 January 2012
} 
studies supporting findings for an association, as reflected in detailed reviews. ${ }^{19}$

In this context, the glucocorticoid-inducible genes Leucin Zipper (GILZ) and serum and glucocorticoid-inducible kinase-1 (SGK-1) are target genes activated by GR activation, and their expression has been shown to be disrupted in the hippocampus as a result of hypercortisolemia. ${ }^{20}$ In addition, a recent study demonstrated a relationship between downregulation of GILZ and an enhanced inflammatory profile in microglia isolated from mice subjected to a chronic stress regimen. ${ }^{21}$ Considering the well-established inverse relationship between GR function and inflammation, ${ }^{4}$ we examined GR expression and expression of the glucocorticoid-inducible genes GILZ and SGK-1 in tandem with inflammatory measures.

The aim of this study was to determine whether glucocorticoid-inducible genes GILZ and SGK-1 and inflammation are related to hippocampal volumes in patients with MDD. Moreover, we wanted to determine how this pathway would be influenced by early childhood adversity.

\section{Participants and methods}

Participants. The study included 40 adult patients with MDD from the mental health services of the Adelaide and Meath Hospital, incorporating the National Children's Hospital, Dublin or St James's Hospital, Dublin. The diagnosis of these patients with MDD was a clinical diagnosis based on DSM-IV criteria and confirmed by an independent psychiatrist using the SCID interview. Forty-three healthy subjects $(\mathrm{HC})$ from the local community were recruited and the groups were balanced for age and sex (Table 1). Fourteen patients received monotherapy with selective serotonin reuptake inhibitor and 15 with a dual acting antidepressant (mirtazapine or venlafaxine). Eleven patients were medication-free when they were transferred to our service and were investigated before restarting antidepressant therapy. Antipsychotics or mood stabilizers were exclusion criteria. Other exclusion criteria were age $<18$ or $>65$, history of neurological or comorbid psychiatric disorders (axis I or axis II), other severe medical illness, head injury or substance abuse. Demographic variables, inclusion and exclusion criteria were documented using a standardized questionnaire and through a structured interview by a psychiatrist.

Written informed consent was obtained from all participants after being given detailed description of the study, which was designed and performed in accordance to the ethical standards laid out by the Declaration of Helsinki and was approved by the ethics committee of St James and the Adelaide and Meath Hospitals, Dublin.

Rating instruments. Self- and observer-rated scales were also filled out for all participants included in the study. The following rating scales were used: the Hamilton Rating Scale for Depression, ${ }^{22}$ Beck's Depression Inventory, ${ }^{23}$ Childhood Trauma Questionnaire (CTQ) ${ }^{24}$ and the Structured Clinical Interview for DSM-IV (SCID-II) personality questionnaire. CTQ is a standardized self-report instrument that assesses five types of childhood maltreatment: emotional, physical, and sexual abuse, and emotional and physical neglect. Reliability and validity of the CTQ have been established, including measures of convergent and discriminative validity from structured interviews, stability over time and corroboration. ${ }^{25}$ Based on the cut-off values for traumatization, we determined participants with and without early childhood adversity. Subjects scoring above the cut-off in at least one of the items were those with childhood adversity. ${ }^{25}$

Magnetic resonance imaging T1 data acquisition. Magnetic resonance images were obtained with a Philips Achieva MRI

Table 1 Demographic data and ELA for patients with MDD and healthy controls

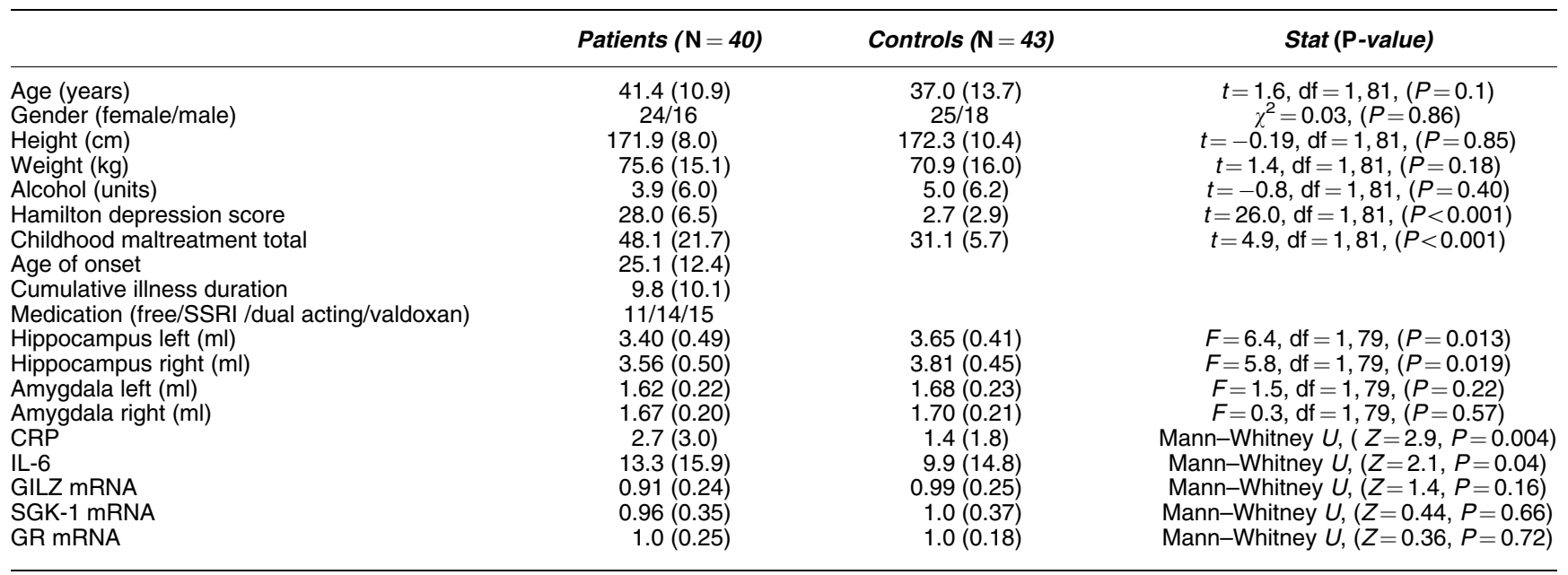

Abbreviations: ANCOVA, analysis of covariance; CRP, C-reactive protein; CTQ, Childhood Trauma Questionnaire; ELA, early life adversity; IL, interleukin; GILZ, glucocorticoid-inducible genes Leucin Zipper; GR, glucocorticoid receptor; MDD, major depressive disorder; SGK-1, serum and glucocorticoid-inducible kinase-1; SSRI, selective serotonin reuptake inhibiter.

Depicted are demographic variables age, gender, height, weight and actual alcohol consumption. Moreover, clinical data depression severity measured with the Hamilton Depression Rating Scale, childhood maltreatment derived as total score from the CTQ, whole hippocampal and amygdala volumes and inflammation markers are indicated for patients and controls. Demographic data were tested by Student's $t$-test (gender with $\chi^{2}$ test), hippocampal and amygdala volumes were subjects to ANCOVA using group as independent factor and age as well as total brain volume as covariates and inflammation markers were tested using Mann-Whitney U-test. 
scanner (Philips Medical System, Netherland BV, Veenphuis 4-6, 5684 PC Best, The Netherlands) operating at 3 Tesla. A sagittal T1 three-dimensional turbo field echo was used to scan all participants (repetition time user defined of $8.5 \mathrm{~ms}$; echo time user defined of $3.9 \mathrm{~ms}$; total acquisition time of $7 \mathrm{~min}$; field of view of foot to head: $256 \mathrm{~mm}$, anterior to posterior: $256 \mathrm{~mm}$, right to left: $160 \mathrm{~mm}$; and a matrix of $256 \times 256$ ). Slice thickness was $1 \mathrm{~mm}$ and voxel size was $1 \times 1 \times 1 \mathrm{~mm}$. All data sets were realigned and resampled three-dimensionally in the anterior commissure to posterior commissure line, according to the coordinates of Talairach, with the software program BRAINS2 (Brain Research: Analysis of Images, Networks and Systems, University of lowa, USA). The program BRAINS2 allowed the regions of interest ${ }^{26}$ to be simultaneously controlled on sagittal, coronal and transverse sections simultaneously.

Definition of hippocampal and amygdala formation. We used manual tracing of the bilateral hippocampal and amygdala structure with the help of the software BRAINS2 as we have previously described (Figure 1). ${ }^{27}$ Additionally, we divided the hippocampal structure into three parts: the head, the body and the tail according to well-established methods. ${ }^{28,29}$ The amygdala tracing is described in detail elsewhere. ${ }^{30}$ The evaluating and tracing researcher (Angela Carballedo for hippocampus, Karim Saleh for amygdala) were blind to participant status. The hippocampus and amygdala were outlined manually using a mouse-driven cursor. To determine inter-rater reliability, 10 brains were randomly chosen and regions of interests determined independently by two raters. The intra-class correlation for inter-rater reliability was high (left hippocampus: ricc $=0.92$, right hippocampus: ricc $=0.88$, left head: ricc $=0.98$, right head: ricc $=0.88$, left body: ricc $=0.87$, right body: ricc $=0.90$, left tail: ricc $=0.78$, right tail: ricc $=0.79$, left amygdala: ricc $=0.85$, right amygdala: ricc $=0.91$ ) (Supplementary Methods).

\section{Measurement of blood inflammatory markers and expression of GR and glucocorticoid-inducible genes}

Blood sampling. A $10 \mathrm{ml}$ blood sample was taken from each subject in the early afternoon before magnetic resonance image scanning into a heparinized vacutainer and was centrifuged to generate plasma. Plasma was stored at $-80^{\circ} \mathrm{C}$ until inflammatory markers were measured. A second blood sample $(2.5 \mathrm{ml})$ was taken into a PAXgene blood RNA tube and used for whole blood RNA isolation and the PAXgene tube was stored at $-20^{\circ} \mathrm{C}$ for $48 \mathrm{~h}$ and then moved to $-80^{\circ} \mathrm{C}$ until RNA extraction was performed. All bloods were taken to the freezer within $1 \mathrm{~h}$ after acquisition. All samples were processed in the same batch with respect to the following methods.

Plasma IL-6 and CRP measurements. Plasma interleukin (IL)-6 was measured using an ELISA MAX Deluxe kit (Biolegend, Cambridge, UK), and plasma CRP concentrations were measured using a CRP ELISA DuoSet (R\&D systems, Abingdon, UK) and these immunoassays were performed according to the manufacturer's instructions.

Real-time PCR analysis of mRNA expression of GR, GILZ and SGK-1 in whole blood samples. RNA isolation was performed using a PAXgene blood RNA kit (Qiagen, West Sussex, UK) and was followed by DNAase treatment to remove contaminating genomic DNA as previously described. ${ }^{31}$ Following RNA quantification and equalization, complementary DNA was synthesized using a cDNA archive kit (Applied Biosystems, Warrington, UK). Gene expression analysis was conducted using real-time PCR employing Taqman Gene Expression Assays (Assay IDs: GR: Hs00353740_m1; GILZ: Hs00608272_m1; SGK-1: Hs00178612_m1; Applied Biosystems) and glyceraldehyde 3-phosphate dehydrogenase served as endogenous control. PCR was performed using ABl's universal cycling conditions on the StepOnePlus Real-Time PCR system (see Supplementary Methods for more detailed information).

Statistics. All statistical analysis were considered to be significant if $P<0.05$. Morphometric measurements in both groups were normally distributed (using Kolmogorov-Smirnov test) and their variances were homogenous (using Levene's test). Correlations between blood markers are indicated in Supplementary Methods 1. We explored associations between CRP and IL-6 with the other variables using regression analysis with $\mathrm{CRP}, \mathrm{IL}-6$ as dependent variables and age, diagnostic group, GR mRNA, GILZ mRNA, SGK-1 mRNA and early life adversity as independent factors.

Hippocampal volumes were subjected to an omnibus analysis of covariance (ANCOVA) to assess the main and interaction effects of the within-subjects factor hemisphere
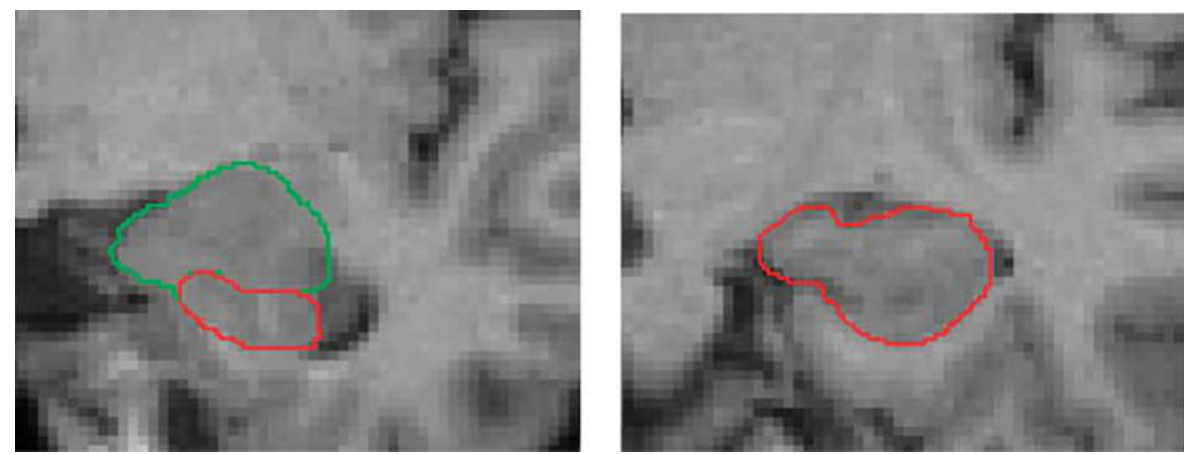

Figure 1 Hippocampus and amygdala tracing: hippocampal head is delineated from the amygdala. 
(left, right) and subregion (head, body, tail), and the betweensubjects factor diagnostic group (MDD, healthy controls $(\mathrm{HC})$ ) and GILZ mRNA expression (low GILZ mRNA, high GILZ $m R N A)$. Left and right amygdala volumes were subjected to similar ANCOVAs without the factor subregions. In the same way, ANCOVAs were calculated using SGK-1, GR, IL-6 and CRP instead of GILZ. Significant interactions were further evaluated with SIDAK-Bonferroni post-hoc tests. A median split was used here to obtain low versus high mRNA expression. As this would change a continuous variable into a categorical variable, we also applied regression analysis as described below.

Moreover, to test which of the inflammation factors or glucocorticoid factors influenced hippocampal or amygdala volumes regression analysis was used with hippocampal or amygdala volumes as dependent variables and childhood maltreatment, age, II-6 concentration, GR mRNA expression, SGK-1 (or GILZ) mRNA expression as independent factors. As significant interactive effects were found between group (patients, controls) and SGK-1 and GILZ mRNA expression the regression analysis were also carried out within the group of patients with depression and the group of HC separately.

\section{Results}

Depressed (MDD) patients did not differ in demographic variables age, gender, weight from $\mathrm{HC}$ (Table 1). IL-6 $(Z=2.1$, $P=0.04)$; and $\operatorname{CRP}(Z=2.9, P=0.004)$ concentrations were higher in patients with MDD compared with HC. GR, SGK-1 and GILZ mRNA did not differ between groups.

CRP or IL-6. In the regression analysis, a significant effect was detected for diagnostic group (patients with MDD, HC) on CRP; with patients with MDD showing higher CRP levels compared with controls (Fchange $=5.4$, rsquare $=0.063$, $\mathrm{df}=1,79, P=0.023$ ). Independent of diagnosis there was an additional effect of childhood maltreatment on CRP (Fchange $=4.4, \quad$ rsquarechange $=0.050, \quad \mathrm{df}=1,78$, $P=0.039$ ). Age, GR, GILZ or SGK-1 mRNA did not show significant effects.

For IL-6, no significant effect was seen in the regression analysis for group. However, there was a trend for a significant effect of childhood maltreatment on IL-6 (Fchange $=2.9$, rsquarechange $=0.035, \mathrm{df}=1,78, P=0.09$ ). A further effect was detected for age (Fchange $=2.9$, rsquarechange $=0.1$, $\mathrm{df}=1,76, P=0.041$ ). The variables $\mathrm{GR}$ mRNA or GILZ (SGK-1) mRNA did not show significant effects.

GILZ or SGK-1 mRNA expression. Next, we investigated whether expression of glucocorticoid-inducible genes had a significant effect on hippocampal volumes in an ANOVA with hippocampal volumes as dependent variables and the withinsubjects factors hemisphere (left, right) and subregion (head, body, tail), and the between-subjects groups (patients, controls) and GILZ mRNA (high, low) (SGK-1, GR mRNA, respectively).

Significant smaller hippocampal volumes were found for patients with MDD compared with controls $(F=8.5$, df $=1,77$, $P=0.005)$. No significant effects or interactions were found
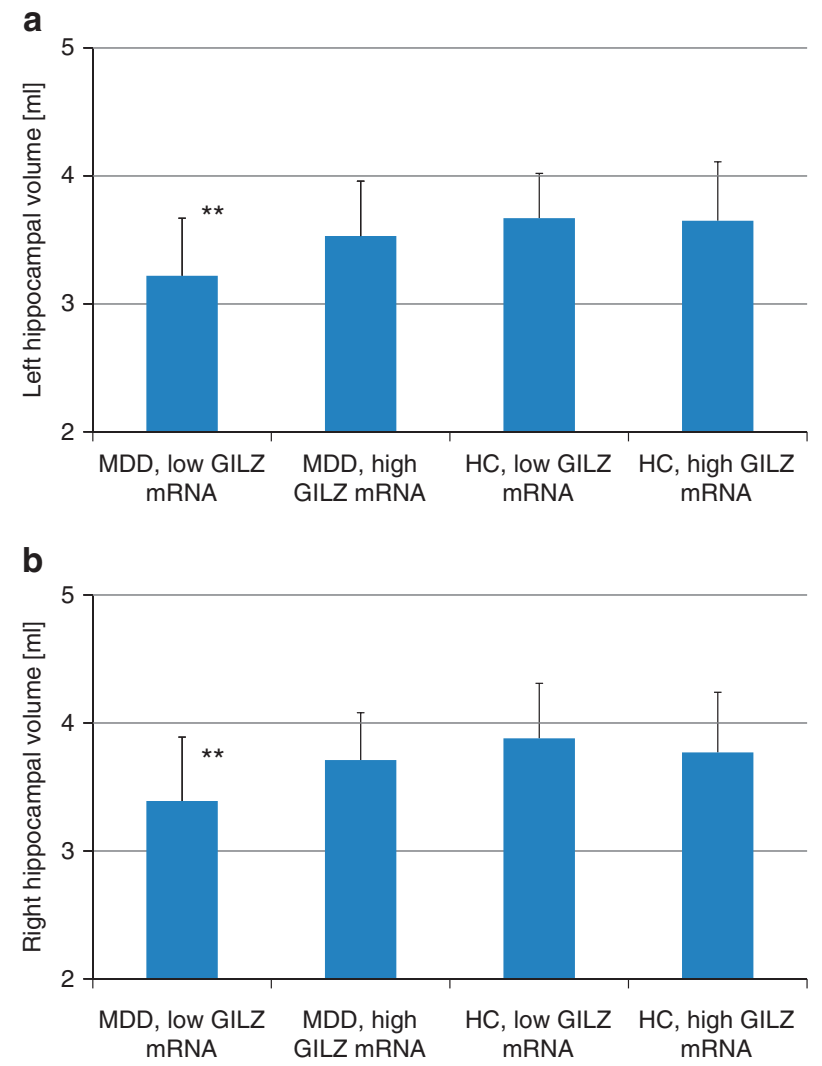

Figure 2 Stratification of patients with major depressive disorder (MDD) and healthy controls $(\mathrm{HC})$ by glucocorticoid-inducible genes Leucin Zipper (GILZ) mRNA. Hippocampal volumes (left (a) and right (b)) are smaller in patients with MDD and low GILZ mRNA concentration compared with $\mathrm{HC}$ with either high or low GILZ mRNA. ${ }^{\star \star} P<0.01$.

for the factors subregions or hemisphere indicating that the significant results apply to the whole hippocampus. There was a significant interaction between diagnostic group and GILZ mRNA (high versus low expression $(F=4.0$, df $=1,77$, $P=0.049)$ ). Post-hoc Sidak-Bonferroni test showed that patients with MDD and low GILZ mRNA concentration had smaller hippocampal volumes than $\mathrm{HC}$ with low GILZ mRNA concentration $(M=-0.47, P=0.003$, Sidak corrected) and $\mathrm{HC}$ with high GILZ mRNA concentration $(M=-0.41$, $P=0.007$, Sidak corrected) (Figure 2).

As GILZ mRNA and SGK-1 mRNA were highly correlated to each other, we also expected these effects in the other mRNAs. A significant interactive effect was also seen for diagnostic groups and SGK-1 mRNA (high versus low expression $(F=4.6, \mathrm{df}=1,77, P=0.035))$. Post-hoc test showed that patients with MDD and low SGK-1 mRNA concentration had smaller hippocampal volumes compared with patients with MDD and high SGK-1 mRNA concentration ( $M=-0.51, P=0.001$, Sidak corrected), HC with low SGK-1 mRNA concentrations $(M=-0.34, P=0.066$, Sidak corrected), and $\mathrm{HC}$ with high SGK-1 mRNA concentrations $(M=-0.42, \quad P=0.006$, Sidak corrected). No significant interaction was found between GR mRNA group and diagnostic group $(F=0.02, \mathrm{df}=1,77, P=0.9)$. 
Amygdala volumes were not significantly different between patients with $\mathrm{MDD}$ and $\mathrm{HC}(F=1.3, \mathrm{df}=1,77, P=0.26)$. There was a trend for larger amygdala volumes in participants with higher GILZ mRNA expression $(F=3.9$, df $=1,77$, $P=0.052)$ or respectively with higher SGK-1 mRNA expression $(F=3.8, \mathrm{df}=1,77, P=0.054)$ compared with those with lower expressions. GR mRNA did not have a significant effect on amygdala volumes $(F=0.07, \mathrm{df}=1,77, P=0.78)$. A significant interactive effect was found between diagnostic group and SGK-1 mRNA expression ( $F=4.2, \mathrm{df}=1,77$, $P=0.043$ ). Post-hoc significant larger amygdala volumes were detected in $\mathrm{HC}$ with high SGK-1 mRNA expression compared with $\mathrm{HC}$ with low SGK-1 mRNA expression $(M=1.7, P=0.029$, Sidak corrected). No significant interaction was found between diagnostic groups and GR mRNA or GILZ mRNA.

Stratification with inflammatory markers. The ANCOVA showed no significant main effect of IL-6 $(F=0.03, \mathrm{df}=1,77$, $P=0.87)$ and no interactive effect between IL-6 and diagnosis $(F=0.29$, df $=1,77, P=0.59)$ on hippocampal volumes. No significant effect was found for CRP $(F=0.08$, df $=1,77$, $P=0.78$ ). No significant interaction was found between diagnostic groups and CRP groups $(F=1.6, \mathrm{df}=1,77$, $P=0.21$ ).

Amygdala volumes were significantly larger in subjects with high IL-6 concentrations compared with those with low IL-6 concentrations $(F=6.2, \mathrm{df}=1,77, P=0.015)$. No effects were found for high versus low CRP concentration $(F=0.5$, $\mathrm{df}=1,77, P=0.48)$. No significant interactions were detected between diagnostic groups and inflammatory profile.
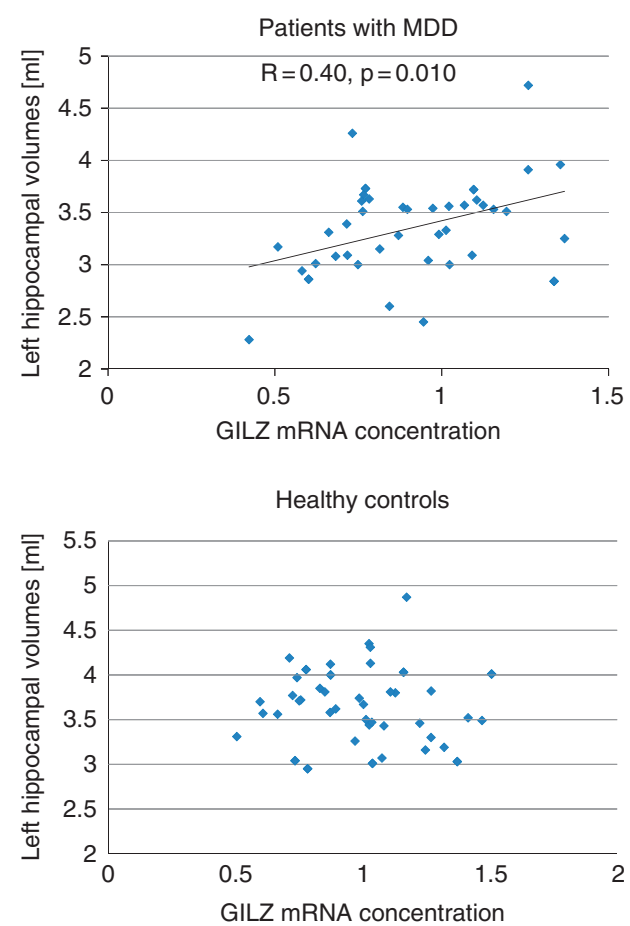

Figure 3 Association between hippocampal volumes and glucocorticoid-inducible genes Leucin Zipper (GILZ) mRNA. Smaller GILZ mRNA concentration is associated with smaller hippocampal volumes in patients with major depressive disorder (MDD) (upper row), but not in healthy controls (lower row).
Regression model. In the whole group of patients and controls, a significant effect of diagnostic group was seen on left hippocampal volumes in the regression analysis (Fchange $=9.9$, rsquarechange $=0.11$, $\mathrm{df}=1,79, P=0.002$ ). GILZ mRNA further showed a trend to influence left hippocampal volumes independent of diagnosis (Fchange $=3.1$, rsquarechange $=0.034, \mathrm{df}=1,78, \quad P=0.08$ ). A significant effect of diagnostic group was also seen on right hippocampal volumes (Fchange $=7.6$, rsquarechange $=0.087$, $\mathrm{df}=1,79, P=0.007)$. GILZ mRNA did not influence right hippocampal volumes further (Fchange $=1.8$, rsquarechange $=0.021$, $\mathrm{df}=1,78, P=0.18$ ). No effect was seen for childhood maltreatment on either right (Fchange $=0.45$, rsquarechange $=0.005, \mathrm{df}=1,77, P=0.50$ ) or left (Fchange $=$ 0.26 , rsquarechange $=0.003, \quad \mathrm{df}=1,77, \quad P=0.61$ ) hippocampal volume or for the variables GR mRNA, IL-6 and CRP.

As there was a significant interactive effect of GILZ (SGK-1 mRNA respectively) with diagnostic groups in the ANOVA, we analyzed the regression model separately within groups. In the patient group, the regression model was significant for the left $(F=2.6, \mathrm{df}=1,38, P=0.037)$ and the right $(F=2.4$, $\mathrm{df}=1,72, P=0.048$ ) hippocampus. The regression analysis found a significant effect of GILZ mRNA expression on left $(\beta=0.59, \quad t=3.4, \quad P=0.002)$ and right $(\beta=0.57, t=3.3$, $P=0.003$ ) hippocampal volumes in the groups of patients (Figure 3, Table 2). IL-6 significantly negatively affected the left $(\beta=-0.44, t=2.1, P=0.044)$ and right $(\beta=-0.44$, $t=2.1, P=0.046$ ) hippocampus. No significant further effects of CRP, age or childhood maltreatment, or GR mRNA were seen. The regression analysis found also a significant effect of
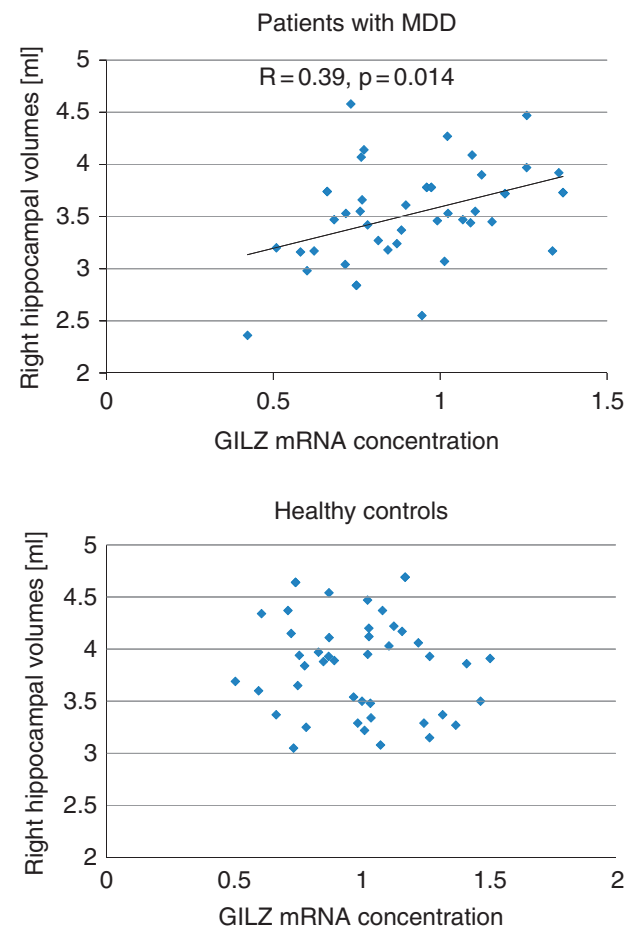
Table 2 Regression analysis for left and right hippocampal volumes as dependent factors and the independent factors GLIZ mRNA, GR mRNA, IL-6, CRP, childhood maltreatment total score and age for patients with MDD and healthy controls

\begin{tabular}{|c|c|c|c|c|c|c|}
\hline & \multicolumn{3}{|c|}{ Left hippocampus } & \multicolumn{3}{|c|}{ Right hippocampus } \\
\hline & Beta & $T$ & P-value & Beta & $T$ & P-value \\
\hline \multicolumn{7}{|l|}{ Patients } \\
\hline GILZ mRNA & 0.59 & 3.4 & 0.002 & 0.57 & 3.3 & 0.003 \\
\hline GR mRNA & -0.05 & -0.29 & 0.77 & -0.03 & -0.16 & 0.87 \\
\hline IL-6 & -0.44 & -2.1 & 0.044 & -0.44 & -2.1 & 0.046 \\
\hline CRP & 0.42 & 2.0 & 0.051 & 0.38 & 1.8 & 0.08 \\
\hline Childhood maltreatment total score & 0.17 & 0.97 & 0.34 & 0.01 & 0.06 & 0.95 \\
\hline Age & 0.14 & 0.81 & 0.43 & 0.24 & 1.3 & 0.20 \\
\hline \multicolumn{7}{|l|}{ Healthy subjects } \\
\hline GILŹ mRNA & -0.13 & -0.62 & 0.54 & -0.16 & -0.75 & 0.46 \\
\hline GR mRNA & 0.08 & 0.34 & 0.74 & 0.03 & 0.15 & 0.89 \\
\hline IL-6 & 0.05 & 0.30 & 0.77 & -0.16 & -0.89 & 0.38 \\
\hline CRP & -0.17 & 1.0 & 0.32 & 0.38 & 1.8 & 0.08 \\
\hline CTQ total score & 0.07 & 0.40 & 0.69 & 0.02 & 0.11 & 0.91 \\
\hline Age & -0.15 & 0.80 & 0.43 & -0.21 & -1.2 & 0.25 \\
\hline
\end{tabular}

Abbreviations: CRP, C-reactive protein; CTQ, Childhood Trauma Questionnaire; IL, interleukin; GILZ, glucocorticoid-inducible genes Leucin Zipper; GR, glucocorticoid receptor; MDD, major depressive disorder.

SGK-1 mRNA expression on left ( $\beta=0.46, t=2.6, P=0.014$ ) and right ( $\beta=0.44, t=2.5, P=0.017$ ) hippocampal volumes in the groups of patients.

In the healthy group, no significant associations were detected between inflammatory factors, age or GR mRNA expression.

There was not a significant effect of any of these variables on amygdala volumes in the regression analysis.

Medication effect. Including medication status (none, selective serotonin reuptake inhibitors, dual) in the analysis did not change the results. There was no significant effect of medication status on hippocampal volumes $(F=0.94$, $\mathrm{df}=2,37, \quad P=0.40)$, on amygdala volumes $(F=0.49$, $\mathrm{df}=2,37, P=0.62), \mathrm{CRP}(F=1.9, \mathrm{df}=2,37, P=0.16)$, IL-6 $(F=0.53, \quad \mathrm{df}=2,37, \quad P=0.59)$, GILZ mRNA $(F=0.28$, $\mathrm{df}=2,37, P=0.76)$ or SGK1 mRNA $(F=1.9, \mathrm{df}=2,37$, $P=0.16)$.

\section{Discussion}

This is the first study that sets the inflammatory state, as assessed by measuring concentrations of CRP and the pro-inflammatory cytokine IL-6, and glucocorticoid status measured using mRNA expression of the GR and the glucocorticoid-inducible genes (GILZ and SGK-1) as well as hippocampus and amygdala into a cohesive pathophysiological human model. There are several novel and remarkable findings that should be highlighted. The smaller hippocampal volumes in patients with MDD were mainly seen in those patients who had less expression of GILZ mRNA, or SGK-1 mRNA respectively, as a marker of reduced activation of the glucocorticoid system compared with those with higher GILZ mRNA expression. In the patients, GILZ and SGK-1 mRNA was positively correlated with hippocampal volumes indicating that in depression the status of the glucocorticoid system may be coupled with hippocampal volumes.

It is noteworthy that a recent study in mice showed that repeated social defeat stress reduced mRNA expression of the glucocorticoid-responsive genes including GILZ in microglia ${ }^{21}$ indicating the involvement of GILZ in stressrelated diseases like depression or anxiety disorders. Moreover, it has been suggested that glucocorticoid resistance may contribute to the inflammatory profile observed in MDD. ${ }^{32}$ In this study, however, we could not find an association between GILZ or SGK-1 mRNA expression and inflammatory state.

Nevertheless, the glucocorticoid system has a central role in the pathophysiology of MDD and there is mounting evidence that specific neuronal circuits, particularly in the developing brain, are damaged by environmental stress inducing changes in the hypothalamic-pituitary-adrenal axis and inflammatory pathways. ${ }^{33}$ Stress-related hypercortisolemia leads to central downregulation of $\mathrm{GRs}^{34}$ and also to glucocorticoid resistance in peripheral immune cells. ${ }^{32}$ In this study, we found evidence that reduced expression of glucocorticoid-inducible genes in patients with MDD, suggesting glucocorticoid resistance, are associated with smaller hippocampal volumes. Such an association might point toward a long-term effect during life, which then might result in smaller hippocampal volumes and glucocorticoid resistance. Thus, both the small hippocampal volume and the low expression of glucocorticoid-inducible genes might be a correlate of vulnerability for depression and this would be in line with smaller hippocampal volumes in subjects at risk for depression. ${ }^{35}$

This is in line with experimental animal and human studies showing the negative effects of chronic exposure to stress and/or adversity on the developing brain. In animal studies, stress leads to reductions in the total volume of the hippocampus, atrophy and loss of neurons in the hippocampus. ${ }^{36}$ A recent study in a sample of patients with first onset psychosis reported that increased IL-6 expression, and increased salivary cortisol levels predicted smaller hippocampal volumes; and that a history of childhood maltreatment was related to current inflammatory markers. ${ }^{37}$

Increased circulating concentrations of $\mathrm{CRP}^{6}$ and inflammatory cytokines such as IL- $6^{5}$ are well established in MDD 
and this could also be confirmed in this study. Moreover, we could show that childhood maltreatment significantly predicts higher CRP levels later in life and has a tendency to predict higher IL-6 levels. These findings are in line with previous studies: childhood maltreatment was positively associated with overall change in plasma IL- 6 response, as well as the maximum IL- 6 concentration during the Trier Social Stress Test (TSST) in 69 healthy adults ${ }^{38}$ and with the CRP concentration in a life-course study cohort of 972 participants. ${ }^{39}$ Moreover, male patients with increased early life stress had higher IL-6 responses to the TSST. ${ }^{40}$ Thus, it was also interesting to look at the association between these inflammatory proteins and hippocampal and amygdala volumes. In the regression model, we found that increased IL-6 plasma concentration was associated with smaller hippocampal volumes, which underlines the role of inflammation in the model of stress-toxicity also in humans.

Recently, along with others, we reported that childhood maltreatment negatively affects hippocampal volumes in MDD. ${ }^{41-44}$ In this study, we could show that childhood maltreatment is associated with increased inflammation, which, in turn, is associated with smaller hippocampal volumes. The fact that childhood maltreatment does not directly predict hippocampal volumes, is not in contradiction to a hypothetical model in which childhood maltreatment might result in higher inflammation and glucocorticoid dysregulation, which then might result in smaller hippocampal volumes over time. As the effects of GILZ and SGK-1 mRNA expression on hippocampal volumes were seen in the patient group only, likely other current or vulnerability factors, for example, like genetic factors, associated with depression might be important additional factors to consider that we did not analyze in this study. Environmental factors like stress and genetic variation are linked together via epigenetic processes. Patients with MDD show smaller hippocampal volumes when they had a history of early life adversity and when they carried the short (S) risk allele of a serotonin transporter variant (5-HTTLPR). ${ }^{45}$

The amygdala was not different between patients with MDD compared with HC. Subsequently, the mix in depressed samples with regards to inflammation and cortisol dysregulation could be the reason for inconsistent findings with respect to amygdala volumes leading to no significant differences in the aggregate. ${ }^{46}$ Higher IL-6 concentrations and higher expression of SGK-1 were associated with larger amygdala volumes.

To overcome the issue of multiple testing, we used omnibus ANOVA designs followed by post-hoc Sidak-Bonferroni tests for multiple testing. Also the sample size with 40 patients and 43 controls was reasonable for an imaging study looking at objective correlations with blood markers, for more in detail interactive analyses between diagnostic groups, glucocorticoid profile and childhood maltreatment a larger group size would have been preferable. A limitation might be that $2 / 3$ of our patients were currently on antidepressant medication, and the other third came to our service medication-free and were scanned before a treatment was initiated. However, the results did not change, when medication status was used as a covariate in the analysis and actual medication status did not have an effect on hippocampal volumes or glucocorticoid or inflammation markers. Retrospective assessment of childhood adversity could be problematic because studies show that usually childhood trauma remains underreported. ${ }^{47}$ We cannot exclude that current depressive symptoms might result in a negative processing bias, however, since our patients with depression were not psychotic or otherwise cognitively disturbed this seems to be rather unlikely.

Based on these findings, we suggest that reduced expression of glucocorticoid-responsive genes like GILZ as well as increased inflammation may have a role in the neuroplasticity-neurotoxicity cascade. Based on our findings, we could argue that reduced glucocorticoid responsiveness and increased inflammation seem to be associated with reduced hippocampal volumes in patients with MDD and that this might form a subgroup of patients who could benefit from therapeutic strategies preventing stress-related neuronal change, for example, $\beta$-adrenergic receptor antagonism prevented the stress-induced changes in microglia and macrophages in areas like the hippocampus. ${ }^{21}$ The present finding strengthens evidence that peripheral inflammatory measures and peripheral measures of GR expression/ function can provide useful information regarding events occurring in the central nervous system. ${ }^{48}$

\section{Conflict of interest}

The authors declare no conflict of interest.

Acknowledgements. We thank Science Foundation Ireland for a fund to conduct the present research to TF (Grant number: SFI/07/SK/B1214C Science Foundation Strokes Professorship Grant). This work was also supported by a TCD School of Medicine Translational Neuroscience grant awarded to TJC and DMcL. We also acknowledge the Health Research Board (HRB) Ireland for funding the Centre for Advanced Medical Imaging (CAMI), St James Hospital.

1. Prince M, Patel V, Saxena S, Maj M, Maselko J, Phillips MR et al. No health without mental health. Lancet 2007; 370: 859-877.

2. Duman RS. Pathophysiology of depression: the concept of synaptic plasticity. Eur Psychiatry 2002; 17(Suppl 3): 306-310.

3. Vreeburg SA, Hoogendijk WJ, van Pelt J, Derijk RH, Verhagen JC, van Dyck R et al. Major depressive disorder and hypothalamic-pituitary-adrenal axis activity: results from a large cohort study. Arch Gen Psychiatry 2009; 66: 617-626.

4. Pace TW, Miller AH. Cytokines and glucocorticoid receptor signaling. Relevance to major depression. Ann NY Acad Sci 2009; 1179: 86-105

5. Simon NM, McNamara K, Chow CW, Maser RS, Papakostas Gl, Pollack MH et al. A detailed examination of cytokine abnormalities in major depressive disorder. Eur Neuropsychopharmacol 2008; 18: 230-233.

6. Howren MB, Lamkin DM, Suls J. Associations of depression with C-reactive protein, IL-1, and IL-6: a meta-analysis. Psychosom Med 2009; 71: 171-186.

7. MacQueen G, Frodl T. The hippocampus in major depression: evidence for the convergence of the bench and bedside in psychiatric research? Mol Psychiatry 2011; 16 : 252-264.

8. Frodl T, Moller HJ, Meisenzahl E. Neuroimaging genetics: new perspectives in research on major depression? Acta Psychiatr Scand 2008; 118: 363-372.

9. Lupien SJ, de Leon M, de Santi S, Convit A, Tarshish C, Nair NP et al. Cortisol levels during human aging predict hippocampal atrophy and memory deficits. Nat Neurosci 1998; 1: 69-73.

10. Knoops AJ, Gerritsen L, van der Graaf Y, Mali WP, Geerlings MI. Basal hypothalamic pituitary adrenal axis activity and hippocampal volumes: the SMART-Medea study. Biol Psychiatry 2010; 67: 1191-1198.

11. Gold SM, Kern KC, O'Connor MF, Montag MJ, Kim A, Yoo YS et al. Smaller cornu ammonis 2-3/dentate gyrus volumes and elevated cortisol in multiple sclerosis patients with depressive symptoms. Biol Psychiatry 2010; 68: 553-559.

12. Mondelli V, Pariante CM, Navari S, Aas M, D'Albenzio A, Di Forti M et al. Higher cortisol levels are associated with smaller left hippocampal volume in first-episode psychosis. Schizophr Res 2010; 119: 75-78. 
13. Bruehl $\mathrm{H}$, Wolf OT, Convit A. A blunted cortisol awakening response and hippocampal atrophy in type 2 diabetes mellitus. Psychoneuroendocrinology 2009; 34: 815-821.

14. Pruessner M, Pruessner JC, Hellhammer DH, Bruce Pike G, Lupien SJ. The associations among hippocampal volume, cortisol reactivity, and memory performance in healthy young men. Psychiatry Res 2007; 155: 1-10.

15. Lindauer RJ, Olff M, van Meijel EP, Carlier IV, Gersons BP. Cortisol, learning, memory, and attention in relation to smaller hippocampal volume in police officers with posttraumatic stress disorder. Biol Psychiatry 2006; 59: 171-177.

16. Dedovic K, Engert V, Duchesne A, Lue SD, Andrews J, Efanov SI et al. Cortisol awakening response and hippocampal volume: vulnerability for major depressive disorder? Biol Psychiatry 2010; 68: 847-853.

17. Colla M, Kronenberg G, Deuschle M, Meichel K, Hagen T, Bohrer M et al. Hippocampal volume reduction and HPA-system activity in major depression. J Psychiatr Res 2007; 41: 553-560.

18. Gunduz-Bruce H, Szeszko PR, Gueorguieva R, Ashtari M, Robinson DG, Kane JM et al. Cortisol levels in relation to hippocampal sub-regions in subjects with first episode schizophrenia. Schizophr Res 2007; 94: 281-287.

19. Frodl T, O'Keane V. How does the brain deal with stress? A review with focus on major depressive disorder. Neurobiol Disease 2011 (in press).

20. Sarabdjitsingh RA, Isenia S, Polman A, Mijalkovic J, Lachize S, Datson N et al. Disrupted corticosterone pulsatile patterns attenuate responsiveness to glucocorticoid signaling in rat brain. Endocrinology 2010; 151: 1177-1186.

21. Wohleb ES, Hanke ML, Corona AW, Powell ND, Stiner LM, Bailey MT et al. Betaadrenergic receptor antagonism prevents anxiety-like behavior and microglial reactivity induced by repeated social defeat. J Neurosci 2011; 31: 6277-6288.

22. Hamilton M. Standardised assessment and recording of depressive symptoms. Psychiatr Neurol Neurochir 1969; 72: 201-205.

23. Beck AT, Steer RA, Brown GK. BDI-II Manual. Harcourt Brace and Company: San Antonio, TX, 1996.

24. Bernstein DP, Fink L, Handelsman L, Foote J, Lovejoy M, Wenzel K et al. Initial reliability and validity of a new retrospective measure of child abuse and neglect. Am J Psychiatry 1994; 151: 1132-1136.

25. Bernstein DP, Stein JA, Newcomb MD, Walker E, Pogge D, Ahluvalia T et al. Development and validation of a brief screening version of the childhood trauma questionnaire. Child Abuse Negl 2003; 27: 169-190.

26. Jorda S, Rossler U, Broido D. Fine structure of excitons and polariton dispersion in quantum wells. Phys Rev B Condens Matter 1993; 48: 1669-1677.

27. Frodl T, Meisenzahl EM, Zetzsche T, Born C, Groll C, Jager M et al. Hippocampal changes in patients with a first episode of major depression. Am J Psychiatry 2002; 159: 1112-1118.

28. Malykhin NV, Bouchard TP, Ogilvie CJ, Coupland NJ, Seres P, Camicioli R. Threedimensional volumetric analysis and reconstruction of amygdala and hippocampal head, body and tail. Psychiatry Res 2007; 155: 155-165.

29. MacQueen GM, Yucel K, Taylor VH, Macdonald K, Joffe R. Posterior hippocampal volumes are associated with remission rates in patients with major depressive disorder. Biol Psychiatry 2008; 64: 880-883.

30. Frodl T, Meisenzahl EM, Zetzsche T, Born C, Jager M, Groll C et al. Larger amygdala volumes in first depressive episode as compared to recurrent major depression and healthy control subjects. Biol Psychiatry 2003; 53: 338-344.

31. Chai V, Vassilakos A, Lee Y, Wright JA, Young AH. Optimization of the PAXgene blood RNA extraction system for gene expression analysis of clinical samples. J Clin Lab Anal 2005; 19: 182-188

32. Zunszain PA, Anacker C, Cattaneo A, Carvalho LA, Pariante CM. Glucocorticoids, cytokines and brain abnormalities in depression. Prog Neuropsychopharmacol Biol Psychiatry 2011; 35: 722-729.
33. Krishnan V, Nestler EJ. The molecular neurobiology of depression. Nature 2008; 455 894-902.

34. Krishnan V, Nestler EJ. Linking molecules to mood: new insight into the biology of depression. Am J Psychiatry 2010; 167: 1305-1320.

35. Amico F, Meisenzahl E, Koutsouleris N, Reiser M, Moller HJ, Frodl T. Structural MRI correlates for vulnerability and resilience to major depressive disorder. J Psychiatry Neurosci 2011; 36: 15-22.

36. Woolley CS, Gould E, McEwen BS. Exposure to excess glucocorticoids alters dendritic morphology of adult hippocampal pyramidal neurons. Brain Res 1990; 531: 225-231.

37. Mondelli V, Cattaneo A, Murri MB, Di Forti M, Handley R, Hepgul N et al. Stress and inflammation reduce brain-derived neurotrophic factor expression in first-episode psychosis: a pathway to smaller hippocampal volume. J Clin Psychiatry 2011; 72: 1677-1684.

38. Carpenter LL, Gawuga CE, Tyrka AR, Lee JK, Anderson GM, Price LH. Association between plasma IL-6 response to acute stress and early-life adversity in healthy adults. Neuropsychopharmacology 2010; 35: 2617-2623

39. Danese A, Pariante CM, Caspi A, Taylor A, Poulton R. Childhood maltreatment predicts adult inflammation in a life-course study. Proc Natl Acad Sci USA 2007; 104: 1319-1324.

40. Pace TW, Mletzko TC, Alagbe O, Musselman DL, Nemeroff CB, Miller AH et al. Increased stress-induced inflammatory responses in male patients with major depression and increased early life stress. Am J Psychiatry 2006; 163: 1630-1633.

41. Dannlowski U, Stuhrmann A, Beutelmann V, Zwanzger P, Lenzen T, Grotegerd D et al. Limbic scars: long-term consequences of childhood maltreatment revealed by functional and structural magnetic resonance imaging. Biol Psychiatry 2012; 71: 286-293.

42. Edmiston EE, Wang F, Mazure CM, Guiney J, Sinha R, Mayes LC et al. Corticostriatallimbic gray matter morphology in adolescents with self-reported exposure to childhood maltreatment. Arch Pediatr Adolesc Med 2011; 165: 1069-1077.

43. Vythilingam M, Heim C, Newport J, Miller AH, Anderson E, Bronen R et al. Childhood trauma associated with smaller hippocampal volume in women with major depression. $\mathrm{Am}$ J Psychiatry 2002; 159: 2072-2080.

44. Frodl T, Reinhold E, Koutsouleris N, Reiser M, Meisenzahl EM. Interaction of childhood stress with hippocampus and prefrontal cortex volume reduction in major depression. J Psychiatr Res 2010; 44: 799-807.

45. Frodl T, Reinhold E, Koutsouleris N, Donohoe G, Bondy B, Reiser M et al. Childhood stress, serotonin transporter gene and brain structures in major depression. Neuropsychopharmacology 2010; 35: 1383-1390.

46. Kempton MJ, Salvador Z, Munafo MR, Geddes JR, Simmons A, Frangou S et al. Structural neuroimaging studies in major depressive disorder. Meta-analysis and comparison with bipolar disorder. Arch Gen Psychiatry 2011; 68: 675-690.

47. McBeth J, Morris S, Benjamin S, Silman AJ, Macfarlane GJ. Associations between adverse events in childhood and chronic widespread pain in adulthood: are they explained by differential recall? J Rheumatol 2001; 28: 2305-2309.

48. Anisman H. Cascading effects of stressors and inflammatory immune system activation: implications for major depressive disorder. J Psychiatry Neurosci 2009; 34: $4-20$

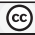

Translational Psychiatry is an open-access journal published by Nature Publishing Group. This work is
licensed under the Creative Commons Attribution-Noncommercial-No Derivative Works 3.0 Unported License. To view a copy of this license, visit http://creativecommons.org/licenses/by-nc-nd/3.0/

\section{Supplementary Information accompanies the paper on the Translational Psychiatry website (http://www.nature.com/tp)}

